PROCEEDINGS OF THE

AMERICAN MATHEMATICAL SOCIETY

Volume 128, Number 6, Pages 1825-1832

S 0002-9939(99)05237-5

Article electronically published on November 29, 1999

\title{
RIGIDITY OF AUTOMORPHISMS AND SPHERICAL CR STRUCTURES
}

\author{
JIH-HSIN CHENG
}

(Communicated by Steven R. Bell)

\begin{abstract}
We establish Bochner-type formulas for operators related to $C R$ automorphisms and spherical $C R$ structures. From such formulas, we draw conclusions about rigidity by making assumptions on the Tanaka-Webster curvature and torsion.
\end{abstract}

\section{Statement of Results}

It is now clear (e.g. [CL1, [CL2, [CT], [Rum]) that certain distinguished secondorder partial differential operators and their fourth-order "Laplacians" play important roles in the study of three-dimensional $C R$ geometry. In this paper we will establish Bochner-type formulas for operators related to $C R$ automorphisms and spherical $C R$ structures. From such formulas, we can draw conclusions about rigidity by making assumptions on the so-called Tanaka-Webster curvature and torsion.

To be precise, let $(M, J, \theta)$ be a smooth, closed (compact without boundary) 3 -dimensional strictly pseudoconvex pseudohermitian manifold (see, e.g. Web, [Le1]). Here $J$ denotes a $C R$ structure and $\theta$ is a contact form, i.e. a nonvanishing real 1-form defining the underlying contact structure. Associated to $(M, J, \theta)$, we have a canonical affine connection and notions of Tanaka-Webster scalar curvature and torsion, denoted $R$ and $A$ (a tensor with coefficient $A_{11}$ or $A_{1}{ }^{\overline{1}}$ ) respectively ([Le1], [Tan], Web]).

Let $T$ be the characteristic vector field of $\theta$ defined by $\theta(T)=1, \mathcal{L}_{T} \theta=0$. (Some authors call $T$ the Reeb vector field.) By choosing suitable complex vector fields $Z_{1}, Z_{\overline{1}}$ such that $J Z_{1}=i Z_{1}, J Z_{\overline{1}}=-i Z_{\overline{1}}$, we form a (unitary) frame $\left\{Z_{1}, Z_{\overline{1}}, T\right\}$ $\left(h_{1 \overline{1}}=1\right)$. The covariant derivatives are taken with respect to this frame and indicated by ${ }_{, 1}, \overline{1},, 0$ and so on. Let $A u t_{0}(J)$ denote the identity component of the $C R$ automorphism group with respect to $J$.

Now we can state our first result.

Theorem A. Let $(M, J, \theta)$ be a smooth, connected, closed 3-dimensional strictly pseudoconvex pseudohermitian manifold.

(a) Suppose $R<0, \sqrt{3} R, 0-2 \operatorname{Im}\left(A_{11, \overline{1} \overline{1}}\right)>0$.

Received by the editors August 7, 1998 .

1991 Mathematics Subject Classification. Primary 32G07; Secondary 32F40, 32C16.

Key words and phrases. Spherical CR structure, Tanaka-Webster curvature, pseudohermitian manifold.

Research supported in part by National Science Council grant NSC 87-2115-M-001-006 (R.O.C.). 
Then $A_{u} t_{0}(J)$ consists of only the identity diffeomorphism.

(b) Suppose $R<0, \sqrt{3} R_{, 0}-2 \operatorname{Im}\left(A_{11, \overline{1} \overline{1}}\right)=0$.

Then $\operatorname{dimAut}(J) \leq 1$.

We remark that the torsion $A=0$ implies the second condition in (b) holds due to the Bianchi identity: $R_{, 0}=A_{11, \overline{1} \overline{1}}+A_{\overline{1} \overline{1}, 11}$. And the result is compatible with Proposition 4.8(a) in $[\mathrm{CT}$. On the other hand, we do not know any examples satisfying curvature conditions in (a).

The idea of proving Theorem A goes as follows. Consider a certain secondorder linear partial differential operator $\mathcal{D}_{J}$ and its "Laplacian" $\mathcal{D}_{J}^{\star} \mathcal{D}_{J}$ acting on functions. Here $\mathcal{D}_{J}^{\star}$ denotes the adjoint of $\mathcal{D}_{J}$. The kernel of $\mathcal{D}_{J}$ parametrizes infinitesimal $C R$ automorphisms (see [CL1] where $\mathcal{D}_{J}$ was denoted $B_{J}^{\prime}$ ). Establish a suitable Bochner-type formula for $\left\langle\mathcal{D}_{J}^{\star} \mathcal{D}_{J} f, f\right\rangle$ by using commutation relations and integration by parts repeatedly (see section 2 for details). Theorem A then follows easily from the final formula. Note that the kernel of $\mathcal{D}_{J}^{\star}$ parametrizes the infinitesimal slice in the study of $C R$ moduli spaces ([CL2]).

Next we consider deformation of spherical $C R$ structures. A $C R$ structure or $C R$ manifold is called spherical if it is locally $C R$-equivalent to the unit sphere with the standard $C R$ structure (e.g. BS, CL1). In dimension 3 it can be characterized quantitatively by the vanishing of a certain fourth-order partial differential operator, the so-called Cartan (curvature) tensor, denoted $Q_{J}$ (while in higher dimensions, Chern's curvature tensor plays the similar role $([\mathrm{CM}])$, which is of second order).

A spherical $C R$ structure $J$ is called rigid if there is no infinitesimal deformation up to diffeomorphisms, i.e. for any smooth family of spherical $C R$ structures $J_{(t)}$ on the base manifold $M$ with $J_{(0)}=J, d /\left.d t\right|_{t=0} J_{(t)}$ equals $\mathcal{L}_{X} J$ for some vector field $X$ of $M$.

To study the rigidity of spherical $C R$ structures, we consider the linearization of $Q_{J}$ plus a symmetry-breaking term provided by $\mathcal{D}_{J} \mathcal{D}_{J}^{\star}$. In section 3 we work out a Bochner-type formula and analyze it to obtain pointwise conditions for $J$ to be rigid.

Theorem B. Let $(M, J)$ be a smooth, closed, spherical CR 3-manifold. Suppose there is a contact form $\theta$ such that $R>0$, (3.11) and (3.12) hold. Then $J$ is rigid.

Note that conditions (a),(b) in Theorem A and (3.11),(3.12) in Theorem B are independent of positive constant multiples of $\theta$. When the torsion vanishes, we have the simplified expression as follows.

Corollary C. Let $(M, J)$ be a smooth, closed, spherical CR 3-manifold. Suppose there is a contact form $\theta$ such that the torsion $A=0$ and

$$
R>0,4 R\left(5 R^{2}+3 \triangle_{b} R\right)-3\left|\nabla_{b} R\right|_{\theta}^{2}>0 .
$$

Then $J$ is rigid.

The sublaplacian and subgradient operators $\triangle_{b}, \nabla_{b}$ acting on (smooth) functions are defined by $\triangle_{b} f=-f_{, 1}{ }^{1}-f_{, 1}{ }^{\overline{1}}$ and $\nabla_{b} f=f,{ }^{1} Z_{1}+f{ }^{\overline{1}} Z_{\overline{1}}$ respectively (cf. [Le1] or [Che]). Also we define $\left|\nabla_{b} f\right|_{\theta}^{2}=2 f_{, 1} f_{,}^{1}$ for real $f$.

Observe that $A=0$ and $R$ being a positive constant satisfy conditions in Corollary C. In this case the universal cover of $(M, J)$ must be compact by Rumin's pseudohermitian version of Myers' theorem ( $\underline{\mathrm{Rum}})$, and hence $C R$-equivalent to 
the standard $S^{3}$. It follows that the fundamental group $\Gamma$ of $M$ is finite. Hence the group cohomology $H^{1}(\Gamma, \mathcal{G})$ in deformation theory (p.232 in BS]) vanishes. So in this special case our result is compatible with the result obtained by "Lie theoretical" argument. Note that a small perturbation of $A=0$ and $R$ being a positive constant still satisfies the conditions in Theorem B.

\section{Proof of Theorem A}

Let $\left\{\theta^{1}, \theta^{\overline{1}}, \theta\right\}$ be the coframe dual to the "unitary" frame $\left\{Z_{1}, Z_{\overline{1}}, T\right\}$ (with $h^{1 \overline{1}}=h_{1 \overline{1}}=1$ in mind, hereafter, we'll write tensors with only lower indices). Recall ([CL2 or [CL1]) that $\mathcal{D}_{J} f=2 R e\left[\left(f_{, 11}+i A_{11} f\right) \theta^{1} \otimes Z_{\overline{1}}\right]$ and the adjoint operator $\mathcal{D}_{J}^{\star}$ acts on a deformation tensor $E=2 \operatorname{Re}\left(E_{11} \theta^{1} \otimes Z_{\overline{1}}\right)$ by

$$
\mathcal{D}_{J}^{\star} E=E_{11, \overline{1} \overline{1}}+i A_{11} E_{\overline{1} \overline{1}}+\text { conjugate. }
$$

Also the generalized Folland-Stein operator $L_{\alpha}$ is defined by $L_{\alpha} f=\triangle_{b} f+i \alpha f_{, 0}$ for a function $f$. By Lemma 2.1 in CL2], we have

$$
\mathcal{D}_{J}^{\star} \mathcal{D}_{J}=(1 / 2) L_{\alpha}^{\star} L_{\alpha}+\mathcal{O}_{2}
$$

with $\alpha=i \sqrt{3}$, where $\mathcal{O}_{2}$ is an operator of weight $\leq 2$. In the rest of this section, we'll look into the details of $\mathcal{O}_{2}$. (Note that $L_{\alpha}$ in the leading term of (2.2) is subelliptic. This implies the existence of the "infinitesimal slice decompositions" in the study of $C R$ moduli spaces without knowing details of the lower-weight term (CL2).)

A direct computation shows that for a real-valued function $f$, we have

$$
\begin{aligned}
& \mathcal{D}_{J}^{\star} \mathcal{D}_{J} f=f_{, 11 \overline{1} \overline{1}}+f_{, \overline{1} \overline{1} 11}+2 \operatorname{Re}\left[2 i A_{11} f_{, \overline{1} \overline{1}}\right. \\
& \left.+2 i A_{11, \overline{1}} f_{, \overline{1}}+i A_{11, \overline{1} \overline{1}} f+\left|A_{11}\right|^{2} f\right] .
\end{aligned}
$$

We'll frequently use the following commutation relations.

Lemma 2.1 (Ricci identities in pseudohermitian geometry). Let $c_{I}$ be a coefficient of some tensor with multi-indices $I$. Suppose $I$ consists of only 1 and $\overline{1}$, and $\alpha=$ (\# of 1 in $I)-(\#$ of $\overline{1}$ in $I)$. Then

$$
\begin{gathered}
c_{I, 1 \overline{1}}-c_{I, \overline{1} 1}=i c_{I, 0}+\alpha c_{I} R, \\
c_{I, 01}-c_{I, 10}=c_{I, \overline{1}} A_{11}-\alpha c_{I} A_{11, \overline{1}}, \\
c_{I, 0 \overline{1}}-c_{I, \overline{1} 0}=c_{I, 1} A_{\overline{1} \overline{1}}+\alpha c_{I} A_{\overline{1} \overline{1}, 1}
\end{gathered}
$$

(this lemma generalizes Lemma 2.3 in Le2] for the three-dimensional case).

By using (2.4),(2.5),(2.6) repeatedly, we obtain

$$
\frac{1}{2} L_{\alpha}^{\star} L_{\alpha} f=f_{, 11 \overline{1} \overline{1}}+f_{, \overline{1} \overline{1} 11}+2 \operatorname{Re}\left[(\sqrt{3}-i)\left(A_{\overline{1} \overline{1}} f_{, 1}\right)_{, 1}-\left(R f_{, 1}\right)_{, \overline{1}}\right]
$$

with the choice of $\alpha=i \sqrt{3}$ eliminating terms having covariant derivative in the direction $T$. Comparing (2.3) with (2.7) and taking the $L^{2}$-inner product with $f$ 
gives

$$
\begin{aligned}
\left\|\mathcal{D}_{J} f\right\|^{2} & =\frac{1}{2}\left\|L_{i \sqrt{3}} f\right\|^{2}-\int_{M} R\left|\nabla_{b} f\right|_{\theta}^{2} d v_{\theta} \\
& +2 \int_{M}\left[\operatorname{Re}\left(\frac{\sqrt{3}+i}{2} A_{11, \overline{1} \overline{1}}\right)+\left|A_{11}\right|^{2}\right] f^{2} d v_{\theta} \\
& +2 \int_{M} \operatorname{Re}\left[(-i-\sqrt{3}) A_{\overline{1} \overline{1}} f_{, 11}\right] f d v_{\theta} .
\end{aligned}
$$

Here the volume form $d v_{\theta}=\theta \wedge d \theta$. With respect to $d v_{\theta}$, we have the divergence theorem and hence integration by parts in calculus of pseudohermitian geometry ([Le2, [Che] ). For instance,

$$
\begin{aligned}
\int_{M} A_{\overline{1} \overline{1}, 1} f_{, 1} \bar{f} d v_{\theta} & =\frac{1}{2} \int_{M} A_{\overline{1} \overline{1}, 1}\left(f^{2}\right)_{, 1} d v_{\theta}(f \text { being real }) \\
& \left.=-\frac{1}{2} \int_{M} A_{\overline{1} \overline{1}, 11} f^{2} d v_{\theta} \text { (integration by parts }\right)
\end{aligned}
$$

was used in deducing (2.8). Now suppose $\phi_{t} \in A u t_{0}(J)$ is a smooth family of $C R$ automorphisms with $\phi_{0}=$ identity. Then $X=\left.\frac{d}{d t}\right|_{t=0} \phi_{t} \in$ Lie $A u t_{0}(J)$ (=Lie algebra of $\left.A_{u t}(J)\right)$ is an infinitesimal $C R$ automorphism, in particular, an infinitesimal contact automorphism. According to Lemma 3.4 and 3.5 in [CL1], $X=X_{f}$ is determined by a function $f=-\theta(X)$ and satisfies the relation: $\mathcal{L}_{X} J=2 \mathcal{D}_{J} f$. Since $\mathcal{L}_{X} J=0$, we get

$$
0=\mathcal{D}_{J} f=f_{, 11}+i A_{11} f
$$

and hence

$$
A_{\overline{1} \overline{1}} f_{, 11}=-i\left|A_{11}\right|^{2} f \text {. }
$$

Substituting (2.9), (2.10) in (2.8), we finally obtain

$$
\begin{aligned}
0 & =\left\|L_{i \sqrt{3}} f\right\|^{2}-2 \int_{M} R\left|\nabla_{b} f\right|_{\theta}^{2} d v_{\theta} \\
& +\int_{M}\left[\sqrt{3} R_{, 0}+i\left(A_{11, \overline{1} \overline{1}}-A_{\overline{1} \overline{1}, 11}\right)\right] f^{2} d v_{\theta} .
\end{aligned}
$$

Now it is easy to see from (2.11) that the condition in (a) of Theorem A implies $f=0$. Therefore $X=X_{f}=0$. For (b), the condition implies $\nabla_{b} f=0$. So $f_{, 0}=0$ by (2.4). Thus $f$ is constant since $M$ is connected. It follows that $\operatorname{dim}\left(\operatorname{Aut}_{0}(J)\right)=$ $\operatorname{dim}\left(\right.$ Lie $\left.\operatorname{Aut}_{0}(J)\right) \leq 1$.

\section{Proof of Theorem B}

Recall ([CL1] $)$ that the Cartan tensor $Q_{J}=i Q_{11} \theta^{1} \otimes Z_{\overline{1}}-i Q_{\overline{1}} \theta^{\overline{1}} \otimes Z_{1}$ where

$$
Q_{11}=\frac{1}{6} R_{, 11}+\frac{i}{2} R A_{11}-A_{11,0}-\frac{2 i}{3} A_{11, \overline{1} 1}
$$

and $J$ is spherical if and only if $Q_{J}=0$. (Note that we have lowered indices using $h_{1 \overline{1}}=1$; also $Q_{J}$ changes "tensorially" when we make a different choice of contact form.) Let $\tilde{J}_{(t)}$ be a smooth family of spherical $C R$ structures with $\tilde{J}_{(0)}=J$. By a theorem of Gray ([Gra or $[\mathrm{Ham})$, there exists a smooth family of diffeomorphisms 
$\phi_{t}$ with $\phi_{0}=$ identity so that, for all $t, J_{(t)}=\phi_{t}^{\star} \tilde{J}_{(t)}$ has the same underlying contact structure as $J$ does. Write the infinitesimal deformation

$$
\left.\frac{d}{d t}\right|_{t=0} J_{(t)}=2 E=4 \operatorname{Re}\left(E_{11} \theta^{1} \otimes Z_{\overline{1}}\right)
$$

and compute $D Q_{J}(2 E)=\left.\partial_{t} Q_{J_{(t)}}\right|_{t=0}$ as we did in [CL1]. There appears a "bad" term $E_{\overline{1} \overline{1}, 1111}$ in the formula, so we add a "symmetry-breaking" term $\mathcal{D}_{J} \mathcal{D}_{J}^{\star} E$ to cancel it. The final formula including terms of lower weights reads

$$
\begin{aligned}
-D & Q_{J}(2 E)+\frac{1}{6} \mathcal{D}_{J} \mathcal{D}_{J}^{\star} E \\
& =2 \operatorname{Re}\left\{\frac{1}{3} E_{11, \overline{1} \overline{1} 11} E_{11,00}-\frac{2 i}{3} E_{11,0 \overline{1} 1}+\frac{i}{3}\left(A_{11} E_{\overline{1} \overline{1}}\right)_{, 11}\right. \\
& -\frac{1}{6} E_{11} R_{, 1 \overline{1}}+\frac{1}{6} E_{11, \overline{1}} R_{, 1}-\frac{1}{6}\left(E_{11} R_{, \overline{1}}\right)_{, 1} \\
& +\frac{1}{2} A_{11}\left(i E_{11, \overline{1} \overline{1}}-i E_{\overline{1} \overline{1}, 11}-A_{11} E_{\overline{1} \overline{1}}-A_{\overline{1} \overline{1}} E_{11}\right)+\frac{i}{2} R E_{11,0} \\
& +2 A_{11}\left(A_{11} E_{\overline{1} \overline{1}}+A_{\overline{1} \overline{1}} E_{11}\right)+\frac{2 i}{3} E_{11} A_{11, \overline{1} \overline{1}}-\frac{2 i}{3} E_{11, \overline{1}} A_{11, \overline{1}}-\frac{2 i}{3}\left(E_{\overline{1} \overline{1}} A_{11,1}\right)_{, 1} \\
& \left.-\frac{4 i}{3}\left(E_{\overline{1} \overline{1}, 1} A_{11}\right)_{, 1}+\frac{i}{6} A_{11}\left(E_{11, \overline{1} \overline{1}}+E_{\overline{1} \overline{1}, 11}+i A_{11} E_{\overline{1} \overline{1}}-i A_{\overline{1} \overline{1}} E_{11}\right)\right\} \theta^{1} \otimes Z_{\overline{1}} .
\end{aligned}
$$

The right-hand side of (3.1) can be written as $\frac{1}{12} L_{\alpha}^{\star} L_{\alpha} E+\mathcal{O}_{2}(E)$ with $\mathcal{O}_{2}$ being an operator of weight $\leq 2$ and $\alpha=4+i \sqrt{3}$. Since $L_{\alpha}$ is subelliptic, the above expression was used in [CL1] to show the short- time solution of a certain regularized evolution equation. Using Lemma 2.1 repeatedly, we can write the highest-weight term of (3.1) as follows:

$$
\begin{aligned}
& \frac{1}{3} E_{11, \overline{1} \overline{1} 11}-E_{11,00}-\frac{2 i}{3} E_{11,0 \overline{1} 1}=\frac{1}{3} E_{11, \overline{1} 1 \overline{1} 1}-E_{11,00}-i E_{11,0 \overline{1} 1} \\
& +\frac{i}{3}\left(E_{11,1} A_{\overline{1} \overline{1}}\right)_{, 1}+\frac{2 i}{3}\left(E_{11} A_{\overline{1} \overline{1}, 1}\right)_{, 1}-\frac{1}{3}\left(R E_{11, \overline{1}}\right)_{, 1} .
\end{aligned}
$$

On the other hand, we compute

$$
\begin{aligned}
& \int_{M} R\left|E_{11,1}\right|^{2} d v_{\theta}=-\int_{M}\left(R E_{11,1 \overline{1}} E_{\overline{1} \overline{1}}+R_{, \overline{1}} E_{11,1} E_{\overline{1} \overline{1}}\right) d v_{\theta} \\
& \text { (by integration by parts) } \\
& \left.=-\int_{M}\left[R\left(E_{11, \overline{1} 1}+i E_{11,0}+2 R E_{11}\right) E_{\overline{1} \overline{1}}+R_{, \overline{1}} E_{11,1} E_{\overline{1} \overline{1}}\right] d v_{\theta} \text { (by }(2.4)\right) .
\end{aligned}
$$

To see how we treat (3.1) in general, we first deal with the torsion $=0$ case. By the local slice theorem ([CL2 $]$ ), there exists a smooth family of contact diffeomorphisms $\psi_{t}$ with $\psi_{0}=$ identity so that $J_{(t)}^{\prime}=\psi_{t}^{\star} J_{(t)}$ lies in the local slice passing through $J$. Since the infinitesimal slice at $J$ is parametrized by the kernel of $\mathcal{D}_{J}^{\star}$, we have $\mathcal{D}_{J}^{\star} E=0$ for $2 E=\left.\frac{d}{d t}\right|_{t=0} J_{(t)}^{\prime}$. 
Now applying (3.1) to such a deformation tensor $E$ : $\mathcal{D}_{J}^{\star} E=0$, substituting (3.2) in (3.1), and taking the $L^{2}$-inner product with $E$, we obtain

$$
\begin{aligned}
0 & =\left\langle-D Q_{J}(2 E), E\right\rangle\left(\text { since } Q_{J_{(t)}^{\prime}}=0\right) \\
& =\int_{M} 2 R e\left\{\frac{1}{3}\left|E_{11, \overline{1} 1}\right|^{2}+\left|E_{11,0}\right|^{2}-i E_{11,0} E_{\overline{1} \overline{1}, 1 \overline{1}}+\frac{1}{6} R\left|E_{11,1}\right|^{2}\right. \\
& +\frac{2 i}{3} R E_{11,0} E_{\overline{1} \overline{1}}-\frac{1}{6} R E_{11, \overline{1} 1} E_{\overline{1} \overline{1}}+\frac{1}{6} R_{, \overline{1}} E_{11,1} E_{\overline{1} \overline{1}} \\
& \left.+\left[\frac{11}{6} R_{, \overline{1} 1}-2 R_{, 1 \overline{1}}+\frac{1}{3} R^{2}\right]\left|E_{11}\right|^{2}\right\} d v_{\theta}
\end{aligned}
$$

by putting $A_{11}=0$, using (3.3) and integration by parts repeatedly.

Note that we reduce (3.4) to the formula (6.3) in [CL1 for $R$ being a constant $\hat{R}$. Furthermore, if $\hat{R}>0$, the right-hand side of (3.4) is a positive definite quadratic hermitian form in $E_{11, \overline{1} 1}, i E_{11,0}$, and $\hat{R} E_{11}$.

It follows that $E=0$ and $0=\left.\frac{d}{d t}\right|_{t=0} J_{(t)}^{\prime}=\left.\frac{d}{d t}\right|_{t=0}\left(\phi_{t} \circ \psi_{t}\right)^{\star} \tilde{J}_{(t)}=\mathcal{L}_{X} J+\left.\frac{d}{d t}\right|_{t=0} \tilde{J}_{(t)}$ where the vector field $X=\left.\frac{d}{d t}\right|_{t=0}\left(\phi_{t} \circ \psi_{t}\right)$. So $J$ is rigid. For general $R$, we require that the integrand in (3.4) is a (pointwise) positive definite quadratic hermitian form in $E_{11, \overline{1} 1}, i E_{11,0}, E_{11,1}, E_{11}$. Now the conditions in Corollary $\mathrm{C}$ can be deduced from basic linear algebra.

When the torsion does not vanish, the formula for $\left\langle-D Q_{J}(2 E), E\right\rangle$ with $\mathcal{D}_{J}^{\star} E=0$ reads

$$
\begin{aligned}
& 0=\left\langle-D Q_{J}(2 E), E\right\rangle=\int_{M}\left\{\frac{2}{3}\left|E_{11, \overline{1} 1}\right|^{2}+2\left|E_{11,0}\right|^{2}\right. \\
& +\frac{1}{3} R\left|E_{11,1}\right|^{2}+\left[\frac{2}{3} R^{2}+\frac{1}{6} \triangle_{b} R+6\left|A_{11}\right|^{2}+\frac{8 i}{3}\left(A_{11, \overline{1} \overline{1}}-A_{\overline{1} \overline{1}, 11}\right)\right]\left|E_{11}\right|^{2} \\
& +2 R e\left[-i E_{11,0} E_{\overline{1} \overline{1}, 1 \overline{1}}+\frac{2 i}{3} R E_{11,0} E_{\overline{1} \overline{1}}-\frac{1}{6} R E_{11, \overline{1} \overline{1}} E_{\overline{1} \overline{1}}\right. \\
& \left.\left.+\left(\frac{1}{6} R_{, \overline{1}}-2 i A_{\overline{1} \overline{1}, 1}\right) E_{11,1} E_{\overline{1} \overline{1}}-\frac{2 i}{3} A_{11,1} E_{\overline{1} \overline{1}, 1} E_{\overline{1} \overline{1}}-\frac{5 i}{3} A_{11} E_{11, \overline{1}} E_{\overline{1} \overline{1}, \overline{1}}\right]\right\} d v_{\theta} .
\end{aligned}
$$

There are non-cross terms like $A_{11,1} E_{\overline{1} \overline{1}, 1} E_{\overline{1} \overline{1}}$ and $A_{11} E_{11, \overline{1}} E_{\overline{1} \overline{1}, \overline{1}}$ in (3.5). We need an inequality to deal with $A_{11} E_{11, \overline{1}} E_{\overline{1} \overline{1}, \overline{1}}$.

Lemma 3.1. Let $\lambda, \rho$ be real numbers. Then

$$
\begin{aligned}
2 \lambda \rho \int_{M} R\left|E_{11, \overline{1}}\right|^{2} d v_{\theta} \leq & \lambda^{2} \int_{M}\left|E_{11, \overline{1} 1}\right|^{2} d v_{\theta}+\rho^{2} \int_{M} R^{2}\left|E_{11}\right|^{2} d v_{\theta} \\
& -\lambda \rho \int_{M}\left(R_{, 1} E_{11, \overline{1}} E_{\overline{1} \overline{1}}+R_{, \overline{1}} E_{\overline{1} \overline{1}, 1} E_{11}\right) d v_{\theta} .
\end{aligned}
$$

For the term $A_{11,1} E_{\overline{1} \overline{1}, 1} E_{\overline{1} \overline{1}}$, we use the following estimate:

$$
2 R e\left(-\frac{2 i}{3} A_{11,1} E_{\overline{1} \overline{1}, 1} E_{\overline{1} \overline{1}}\right) \geq-\frac{2}{3}\left|A_{11,1}\right|^{\frac{2}{3}}\left|E_{\overline{1} \overline{1}, 1}\right|^{2}-\frac{2}{3}\left|A_{11,1}\right|^{\frac{4}{3}}\left|E_{11}\right|^{2} .
$$

(To deduce (3.7), replace $a, b$ by $\omega a, \omega^{2} b$ in the basic inequality $2 R e(a b) \geq-|a|^{2}-|b|^{2}$ with $a=-i E_{\overline{1} \overline{1}, 1}, b=E_{\overline{1} \overline{1}}$, and $\omega^{3}=A_{11,1}$.)

The reason for taking fractional exponents in (3.7) is to make our conditions invariant under the scale change of contact form by a positive constant multiple as we'll see later. Take a small amount of $\int_{M}\left|E_{11, \overline{1} 1}\right|^{2} d v_{\theta}$ and $\int_{M} R^{2}\left|E_{11}\right|^{2} d v_{\theta}$ to gain the term $\int_{M} R\left|E_{11, \overline{1}}\right|^{2} d v_{\theta}$ by (3.6) in the right-hand side of (3.5) while keeping 
the quadratic hermitian form in $E_{11, \overline{1} 1}, i E_{11,0}, E_{11,1}, E_{11, \overline{1}}, E_{11}$ positive definite at least for $R=$ constant $>0, A_{11}=0$. For instance, we can take $\lambda=\rho=\frac{1}{4}$ in (3.6) and then use it and (3.7) in estimating the right-hand side of (3.5). The final result reads

$$
\begin{aligned}
0 \geq & \int_{M}\left\{\frac{29}{48}\left|E_{11, \overline{1} 1}\right|^{2}+2\left|E_{11,0}\right|^{2}+\frac{1}{3} R\left|E_{11,1}\right|^{2}\right. \\
& +\left(\frac{1}{8} R-\frac{2}{3}\left|A_{11,1}\right|^{\frac{2}{3}}\right)\left|E_{11, \overline{1}}\right|^{2}+\left[\frac{29}{48} R^{2}+\frac{11}{48} \triangle_{b} R\right. \\
& \left.+6\left|A_{11}\right|^{2}+\frac{8 i}{3}\left(A_{11, \overline{1} \overline{1}}-A_{\overline{1} \overline{1}, 11}\right)-\frac{2}{3}\left|A_{11,1}\right|^{\frac{4}{3}}\right]\left|E_{11}\right|^{2} \\
& +2 R e\left[-i E_{11,0} E_{\overline{1} \overline{1}, 1 \overline{1}}+\frac{2}{3} i R E_{11,0} E_{\overline{1} \overline{1}}-\frac{1}{6} R E_{11, \overline{1} 1} E_{\overline{1} \overline{1}}\right. \\
& \left.\left.+\left(\frac{5}{48} R_{, \overline{1}}-2 i A_{\overline{1} \overline{1}, 1}\right) E_{11,1} E_{\overline{1} \overline{1}}-\frac{5 i}{3} A_{11} E_{11, \overline{1}} E_{\overline{1} \overline{1}, \overline{1}}\right]\right\} d v_{\theta} .
\end{aligned}
$$

The integrand in (3.8) is a quadratic hermitian form in $E_{11, \overline{1} 1}, i E_{11,0}, E_{11,1}$, $E_{11, \overline{1}}$, and $E_{11}$. By basic linear algebra it is positive definite if and only if $R>0$,

$$
\begin{array}{ccccc} 
& \begin{array}{cccc}
\frac{29}{48} & -1 & 0 & 0 \\
-1 & 2 & 0 & 0 \\
0 & 0 & \frac{1}{3} R & \frac{5 i}{3} A_{\overline{1} \overline{1}} \\
0 & 0 & \frac{-5 i}{3} A_{11} & \frac{1}{8} R-\frac{2}{3}\left|A_{11,1}\right|^{\frac{2}{3}}
\end{array} \mid>0, \text { and } \\
& & & & \\
\frac{29}{48} & -1 & 0 & 0 & -\frac{1}{6} R \\
-1 & 2 & 0 & \frac{5 i}{3} A_{\overline{1} \overline{1}} & \frac{5}{48} R_{, \overline{1}}-2 i A_{\overline{1} \overline{1}, 1} \\
0 & 0 & \frac{1}{3} R & \frac{2}{3} \\
0 & 0 & \frac{-5 i}{3} A_{11} & \frac{1}{8} R-\frac{2}{3}\left|A_{11,1}\right|^{\frac{2}{3}} & \mathcal{M} \\
-\frac{1}{6} R & \frac{2}{3} R & \frac{5}{48} R, 1+2 i A_{11, \overline{1}} & 0 &
\end{array}
$$

is larger than 0 . Here $\mathcal{M}=\frac{29}{48} R^{2}+\frac{11}{48} \triangle_{b} R+6\left|A_{11}\right|^{2}-\frac{2}{3}\left|A_{11,1}\right|^{\frac{4}{3}}+\frac{8 i}{3}\left(A_{11, \overline{1} \overline{1}}-A_{\overline{1} \overline{1}, 11}\right)$. A straightforward computation shows that (3.9) is equivalent to

$$
\frac{3}{8} R^{2}-2 R\left|A_{11,1}\right|^{\frac{2}{3}}-25\left|A_{11}\right|^{2}>0
$$

while (3.10) is equivalent to

$$
\begin{aligned}
& \left(\frac{3}{8} R^{2}-2 R\left|A_{11,1}\right|^{\frac{2}{3}}-25\left|A_{11}\right|^{2}\right)\left\{\frac{83}{3456} R^{2}\right. \\
& \left.+\frac{55}{1152} \triangle_{b} R+\frac{5}{4}\left|A_{11}\right|^{2}-\frac{5}{36}\left|A_{11,1}\right|^{\frac{4}{3}}+\frac{5 i}{9}\left(A_{11, \overline{1} \overline{1}}-A_{\overline{1} \overline{1}, 11}\right)\right\} \\
& -\frac{15}{8}\left(\frac{1}{8} R-\frac{2}{3}\left|A_{11,1}\right|^{\frac{2}{3}}\right)\left|\frac{5}{48} R_{, \overline{1}}-2 i A_{\overline{1} \overline{1}, 1}\right|^{2}>0 .
\end{aligned}
$$

Observe that if $\theta$ changes by a positive constant multiple $k, R$ and $A_{11}$ change by multiplying $k^{-1}$ while $A_{11,1}, A_{\overline{1} 1,1}$, and $R_{, \overline{1}}$ change by multiplying $k^{-\frac{3}{2}}$. Similarly $A_{11, \overline{1} \overline{1}}, A_{\overline{1} \overline{1}, 11}$, and $\triangle_{b} R$ change by multiplying $k^{-2}$. So the conditions (3.11),(3.12) are invariant under the change of contact form by a positive constant multiple. Now Theorem $B$ follows from (3.8) under the conditions (3.11),(3.12). 


\section{ACKNOWLEDGMENT}

This work was carried out during the author's visit at Harvard University. He would therefore like to thank the members of the Mathematics Department, and especially Professor Shing-Tung Yau, for their hospitality during his stay.

\section{REFERENCES}

[BS] D. Burns and S. Shnider, Spherical hypersurfaces in complex manifolds, Invent. Math. 33 (1976),223-246 MR 54:7875]

[Che] J.-H. Cheng, Curvature functions for the sphere in pseudohermitian geometry, Tokyo J. Math. 14(1991), 151-163 MR 92k:32016

[CL1] J.-H. Cheng and J. M. Lee, The Burns-Epstein invariant and deformation of CR structures, Duke Math. J. 60(1990), 221-254 MR 91a:32023

[CL2] A local slice theorem for 3-dimensional CR structures, Amer. J. Math. 117(1995), 12491298 MR 96f:32030

[CM] S.-S. Chern and J. K. Moser, Real hypersurfaces in complex manifolds, Acta Math. 133(1974), 219-271 MR 54:13112

[CT] J.-H. Cheng and I-H. Tsai, Deformation of spherical CR structures and the universal Picard variety, to appear in Communications in Analysis and Geometry

[Gra] J. W. Gray, Some global properties of contact structures, Ann. Math. 69(1959), 421-450 MR 22:3016

[Ham] R. S. Hamilton, The inverse function theorem of Nash and Moser, Bull. Amer. Math. Soc. 7(1982), 65-222 MR 83j:58014

[Le1] J. M. Lee, The Fefferman metric and pseudohermitian invariants, Trans. AMS 296(1986), 411-429 MR 87j:32063

[Le2] J. M. Lee, Pseudo-Einstein structures on CR manifolds, Am. J. Math. 110(1988), 157-178 MR 89f:32034

[Rum] M. Rumin, Formes différentielles sur les variétés de contact, J. Diff. Geom., 39(1994), 281-330 MR 95g:58221

[Tan] N. Tanaka, A Differential Geometric Study on Strongly Pseudo-Convex Manifolds, 1975, Kinokuniya Co. Ltd., Tokyo MR 53:3361

[Web] S. M. Webster, Pseudohermitian structures on a real hypersurface, J. Diff. Geom. 13(1978), 25-41 MR 80e:32015

Institute of Mathematics, Academia Sinica, Taipei, Taiwan

E-mail address: cheng@math.sinica.edu.tw 